\title{
Efeito do uso de lente escleral cosmética na sensibilidade tátil corneal em phthisis bulbi
}

\author{
Effect of ocular prosthesis on cornea sensitivity in phthisis bulbi
}

\author{
Lucia Miriam Dumont Lucci ${ }^{1}$ \\ Cristina Nagako Itami ${ }^{2}$ \\ Rosana Francisco Alves ${ }^{3}$ \\ Fabio Tadeu Montesano ${ }^{4}$ \\ Midori Hentona Osaki² \\ Ana Estela B.P.P.Sant'Anna ${ }^{5}$
}

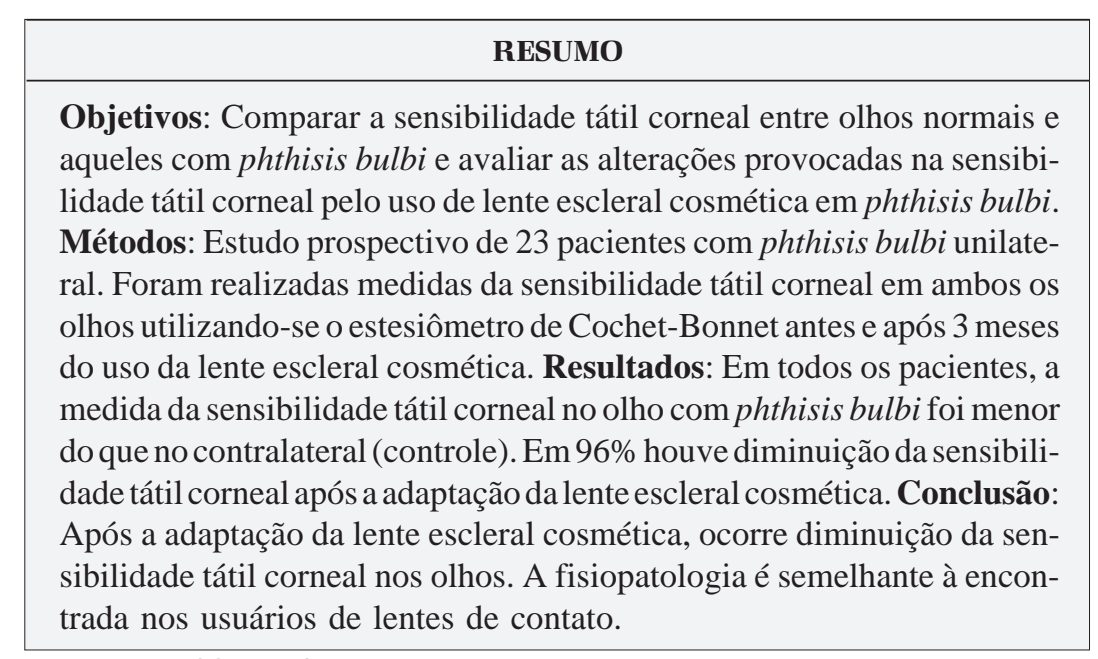

Descritores: Córnea; Olho artificial; Dermatite de contato; Anoxia

\section{INTRODUÇÃO}

Há numerosas terminações nervosas entre as células superficiais do epitélio corneano, o que faz com que esta seja uma das mais sensíveis regiões do corpo humano.

A diminuição da sensibilidade tátil corneal causada por mudanças neurotróficas tem sido bem documentada em pacientes com alterações sistêmicas ou oculares. Alguns exemplos são diabetes mellitus ${ }^{(1)}$, hanseníase ${ }^{(2)}$, usuários de lentes de contato gelatinosas ou gás permeáveis ${ }^{(3-5)}$, cirurgias oculares $^{(6-7)}$, cirurgia refrativa ${ }^{(8)}$ e pacientes com hiposecreção lacrimal ${ }^{(9)}$.

A sensibilidade tátil corneal é um importante mecanismo de defesa do olho e sua diminuição predispõe a córnea a suportar maiores traumas e, nos casos de hanseníase, sem sintomatologia ${ }^{(2)}$. Quantificar a sensibilidade tátil corneal é um exame importante para o diagnóstico diferencial de várias doenças do segmento anterior do bulbo ocular ${ }^{(7)}$.

Em 1960, Cochet e Bonnet descreveram um instrumento para avaliar a sensibilidade tátil corneal ${ }^{(10)}$. O estesiômetro consiste de um monofilamento de náilon de $60 \mathrm{~mm}$ de comprimento e $0,12 \mathrm{~mm}$ de diâmetro, montado em um suporte que permite a variação do comprimento do monofilamento de 5 a $60 \mathrm{~mm}$. Cada determinado comprimento, quando aplicado perpendicularmente à superfície corneana, exerce uma pressão que varia de 11 a $200 \mathrm{mg} / 0,0113 \mathrm{~mm}^{2(10)}$.

Lawrenson e Ruskell, utilizando o estesiômetro de Cochet-Bonnet, observaram que a sensibilidade tátil corneal no limbo temporal é maior do que no limbo inferior ${ }^{(5)}$.

Alguns olhos cegos adquirem um aspecto visual pouco aceitável devi- 
do a hiperemia bulbar e a diminuição do tamanho do bulbo ocular. Muitos pacientes não aceitam a cirurgia para a retirada do globo ocular apesar do olho não ter visão. Nos casos não dolorosos pode-se indicar a adaptação de uma lente escleral cosmética (LEC) para melhorar a aparência estética do paciente e facilitar sua readaptação ao convívio social.

As LEC possuem dimensões superiores as da LC e estão em contato com a córnea e a superfície conjuntival. Como seria a tolerância de uma lente sobre um bulbo ocular anatomicamente alterado? Há diminuição da sensibilidade tátil corneal com o uso prolongado de uma LEC?

Estas dúvidas motivaram a realização deste estudo que teve como objetivos:

1. comparar a sensibilidade tátil corneal de olhos normais e phthisis bulbi.

2. avaliar a alteração na sensibilidade tátil corneal devido o uso de LEC.

\section{MÉTODOS}

Foram estudados prospectivamente 23 pacientes com phthisis bulbi unilateral. Nenhum dos pacientes tinha indicação de evisceração ou enucleação, pois não apresentavam dor ocular, queixando-se apenas do aspecto estético. Em todos os pacientes realizou-se o exame de ultra-sonografia que confirmou a phthisis bulbi através da diminuição do diâmetro antero-posterior do bulbo ocular e espessamento da coróide. As causas de phthisis bulbi encontradas em nossos pacientes foram: trauma perfurante (15 casos), glaucoma congênito (3 casos), descolamento de retina (2 casos), microftalmo congênito ( 2 casos) e origem desconhecida em um paciente. Foram excluídos deste estudo, olhos com alterações corneanas, impossibilidade de realizar a medida da sensibilidade tátil corneal, portadores de doenças que poderiam causar diminuição da sensibilidade tátil corneal, usuários de lentes de contato (LC), história de ceratite por herpes e pós-cirurgia refrativa.

Antes da adaptação da LEC, os pacientes foram esclarecidos quanto aos objetivos deste estudo e foram submetidos ao exame de sensibilidade tátil corneal bilateral utilizando-se o estesiômetro de Cochet-Bonnet (Luneau Ophthalmologie, Paris, França). Os pacientes foram examinados em decúbito dorsal horizontal e em posição primária do olhar, tomando-se o cuidado para se evitar a circulação de ar na sala.

A sensibilidade tátil foi determinada pela pressão exercida pelo monofilamento de náilon, colocado em contato perpendicular à córnea, a $2 \mathrm{~mm}$ do limbo temporal. Iniciava-se o exame com a menor pressão e continuava de modo progressivo até o paciente piscar ou informar a sensação de toque do monofilamento.

A tabela 1 mostra o valor da pressão exercida pelo monofilamento de náilon $\left(\mathrm{mg} / 0,0113 \mathrm{~mm}^{2}\right)$ conforme o comprimento do mesmo.

A sensibilidade tátil corneal foi medida sempre nos dois olhos, iniciando-se o exame pelo olho normal. A primeira medida da sensibilidade tátil corneal foi realizada previamente à adaptação da LEC e a segunda medida foi realizada 3 meses após adaptação. O seguimento destes pacientes variou de 3 a 6 meses do uso da LEC.

A análise estatística foi feita com o teste de $t$ de Student para amostras relacionadas, no qual comparamos a sensibilidade tátil corneal média dos olhos normais com phthisis bulbi antes da adaptação da LEC, olhos normais com phthisis bulbi após LEC e phthisis bulbi antes e após a adaptação da LEC. O nível de significância foi de $0,05^{(11)}$. O gráfico feito foi do tipo "box-plot".

Definimos como comparação 1, as medidas da sensibilidade tátil corneal do olho normal e phthisis bulbi antes da adaptação da LEC. A comparação 2 foi realizada 3 meses após a adaptação da LEC e a terceira comparação consiste na diferença da sensibilidade tátil corneal dos olhos em phthisis bulbi antes e depois da adaptação da LEC.

\section{RESULTADOS}

Foram estudados 23 pacientes com idades entre 7 e 45 anos (média 26 anos) e $70 \%$ eram do sexo masculino. Dos pacientes estudados, $60 \%$ tinham o olho esquerdo acometido.

A estesiometria nos olhos normais variou de 4,5 a $6,0 \mathrm{~cm}$ (média 5,8 cm) e nos olhos com atrofia ocular de 1,0 a 5,5 cm (média 2,6 cm). Após 3 meses do uso da LEC, o grupo dos olhos normais não mostrou alteração na sensibilidade tátil corneal enquanto o grupo de phthisis bulbi mostrou uma variação de 0,5 a 5,0 cm (média 1,5 cm) (Tabela 2).

Em todos os pacientes, a medida da sensibilidade tátil corneal do olho em phthisis bulbi foi menor do que a do olho normal (controle), pré e pós-adaptação da LEC.

Com relação aos olhos em phthisis bulbi, 22 pacientes (96\%) apresentaram diminuição da sensibilidade tátil corneal após a adaptação da LEC.

Quatro pacientes (casos: 9, 10, 14 e 16) apresentavam no olho em phthisis bulbi uma sensibilidade tátil corneal alta (maior que 3,0 cm) após a adaptação da LEC. Em 3 destes (casos: 9, 10 e 16) foi realizada a avaliação da sensibilidade tátil

\begin{tabular}{|c|c|}
\hline $\begin{array}{l}\text { Comprimento do } \\
\text { náilon }(\mathrm{cm})\end{array}$ & $\begin{array}{c}\text { Valores de pressão } \\
\left(\mathrm{mg} / 0,0113 \mathrm{~mm}^{2}\right)\end{array}$ \\
\hline 6,0 & 11 \\
\hline 5,5 & 12 \\
\hline 5,0 & 13 \\
\hline 4,5 & 16 \\
\hline 4,0 & 21 \\
\hline 3,5 & 27 \\
\hline 3,0 & 36 \\
\hline 2,5 & 52 \\
\hline 2,0 & 75 \\
\hline 1,5 & 100 \\
\hline 1,0 & 145 \\
\hline 0,5 & 200 \\
\hline
\end{tabular}




\begin{tabular}{|c|c|c|c|c|c|c|}
\hline & \multicolumn{2}{|c|}{ Pré adaptação } & \multicolumn{2}{|c|}{ Pós adaptação } & \multicolumn{2}{|c|}{6 meses } \\
\hline & Olho normal & Phthisis bulbi & Olho normal & Phthisis bulbi & Olho normal & Phthisis bulbi \\
\hline 1 & 5,5 & $3,5 /$ od & 5,5 & 2,0 & & \\
\hline 2 & 6,0 & $1,0 /$ oe & 6,0 & 0,5 & & \\
\hline 3 & 6,0 & $5,0 /$ od & 6,0 & 1,5 & & \\
\hline 4 & 6,0 & $1,5 /$ od & 6,0 & 1,0 & & \\
\hline 5 & 6,0 & $2,0 /$ oe & 6,0 & 0,5 & & \\
\hline 6 & 6,0 & $2,5 /$ oe & 6,0 & 1,5 & & \\
\hline 7 & 6,0 & $3,5 /$ oe & 6,0 & 0,5 & & \\
\hline 8 & 6,0 & $2,0 /$ oe & 6,0 & 1,0 & & \\
\hline 9 & 6,0 & $4,5 /$ oe & 6,0 & 4,5 & 6,0 & 4,0 \\
\hline 10 & 6,0 & $5,5 /$ od & 6,0 & 5,0 & 6,0 & 4,5 \\
\hline 11 & 6,0 & $1,5 /$ od & 6,0 & 0,5 & & \\
\hline 12 & 5,5 & $2,5 /$ od & 5,0 & 1,5 & & \\
\hline 13 & 5,5 & $4,5 /$ oe & 5,5 & 1,5 & & \\
\hline 14 & 6,0 & $5,0 /$ od & 6,0 & 3,5 & & \\
\hline 15 & 4,5 & $1,5 /$ oe & 4,5 & 0,5 & & \\
\hline 16 & 6,0 & $3,5 /$ oe & 6,0 & 3,0 & 6,0 & 2,0 \\
\hline 17 & 6,0 & $2,5 /$ oe & 6,0 & 1,0 & & \\
\hline 18 & 6,0 & $2,0 /$ oe & 6,0 & 1,5 & & \\
\hline 19 & 6,0 & $1,0 /$ od & 6,0 & 0,5 & & \\
\hline 20 & 6,0 & $2,0 /$ od & 6,0 & 1,5 & & \\
\hline 21 & 5,5 & $1,0 /$ oe & 5,5 & 0,5 & & \\
\hline 22 & 6,0 & $1,5 /$ oe & 6,0 & 1,0 & & \\
\hline 23 & 6,0 & $1,0 /$ od & 6,0 & 0,5 & & \\
\hline
\end{tabular}

corneal no sexto mês do uso da LEC e em todos houve uma diminuição da mesma, embora tenha variado pouco nos pacientes 9 e 10 (Tabela 2). Não foi possível avaliar o paciente 14 no sexto mês de uso da LEC, pois o paciente retornou à sua cidade de origem.

Observou-se que a média de estesiometria no grupo préadaptado é estatisticamente maior em olhos normais do que em phthisis bulbi, variando de 2,6 a 3,8 cm (p<0,001). O mesmo foi observado no pós-adaptado, variando de 3,7 a 4,9 (p<0,001) e a média de estesiometria nos olhos em phthisis bulbi é estatisticamente maior no pré-adaptado do que no pós-adaptado, variando de 0,7 a 1,5 (p<0,001) (Gráfico 1 e Tabela 3).

\section{DISCUSSÃO}

A adaptação de LEC constitui uma opção conservadora, simples, rápida e com um resultado estético satisfatório.

A medida da sensibilidade tátil corneal com o estesiômetro de Cochet-Bonnet torna o estudo confiável e reprodutível. Estudos observaram que a sensibilidade tátil corneal do limbo temporal é estatisticamente maior do que a do limbo inferior ${ }^{(5)}$, razão pela qual avaliamos a sensibilidade tátil corneal no limbo temporal em todos os olhos deste estudo.

Lentes de contato (LC) de polimetilmetacrilato causam importante redução na sensibilidade tátil corneal ${ }^{(3)}$. A diminuição da sensibilidade tátil corneal ocorre exponencialmente com a dura- ção do uso da LC. Pacientes usuários de LC há 1-2 anos apresentam sensibilidade tátil corneal semelhante ao grupo controle (média 3,5 cm), porém nos usuários de LC há 17-22 anos a sensibilidade tátil corneal média é de $1,5 \mathrm{~cm}^{(3)}$. Neste estudo, nos três pacientes com seguimento maior (6 me-ses), observou-se a diminuição progressiva da sensibilidade tátil corneal.

Apesar do processo de diminuição da sensibilidade tátil corneal ser desconhecido, sabe-se que diminui exponencialmente em função da duração do uso das lentes ${ }^{(3)}$. Com o uso das LC, as terminações nervosas livres não são atrofiadas, mas ficam quiescentes. Além disso, a pressão exercida pelas LC na córnea com o piscar é significativa(3).

As terminações nervosas são muito numerosas entre as células superficiais do epitélio corneal, tornando a córnea um dos tecidos mais sensíveis do organismo ${ }^{(4)}$.

A avaliação da sensibilidade tátil corneal é muito importante na adaptação de LC e LEC. Sensibilidade tátil corneal muito baixa constitui uma contra-indicação para adaptação de lente de contato, já que aumenta o número de complicações. Ao contrário, hipersensibilidade implica numa baixa tolerância na adaptação de lente de contato.

Vários estudos mostram diminuição da sensibilidade tátil corneal significantemente nos usuários de LC gelatinosas e proporcional ao conteúdo hídrico da LC. Velasco et al observaram uma diminuição da sensibilidade tátil corneal significantemente maior nos usuários de LC gelatinosa com $38 \%$ de conteúdo hídrico em relação a 55\%. Isto reflete a alteração 


\begin{tabular}{|c|c|c|c|c|c|c|c|c|}
\hline \multirow[b]{2}{*}{ Diferenças } & \multirow[b]{2}{*}{$\mathbf{N}$} & \multirow[b]{2}{*}{ Média } & \multirow[b]{2}{*}{ Desvio padrão } & \multirow[b]{2}{*}{ Mínimo } & \multirow[b]{2}{*}{ Máximo } & \multicolumn{2}{|c|}{ Intervalo de confiança } & \multirow[b]{2}{*}{$\mathbf{P}$} \\
\hline & & & & & & Limite inferior & Limite superior & \\
\hline Pré N-AN & 23 & 3,2 & 1,5 & 1,0 & 5,0 & 2,6 & 3,8 & $<0,001$ \\
\hline PósN-AN & 23 & 4,3 & 1,3 & 1,0 & 6,0 & 3,7 & 4,9 & $<0,001$ \\
\hline ANPré-ANPós & 23 & 1,1 & 0,9 & 0,0 & 4,0 & 0,7 & 1,5 & $<0,001$ \\
\hline
\end{tabular}

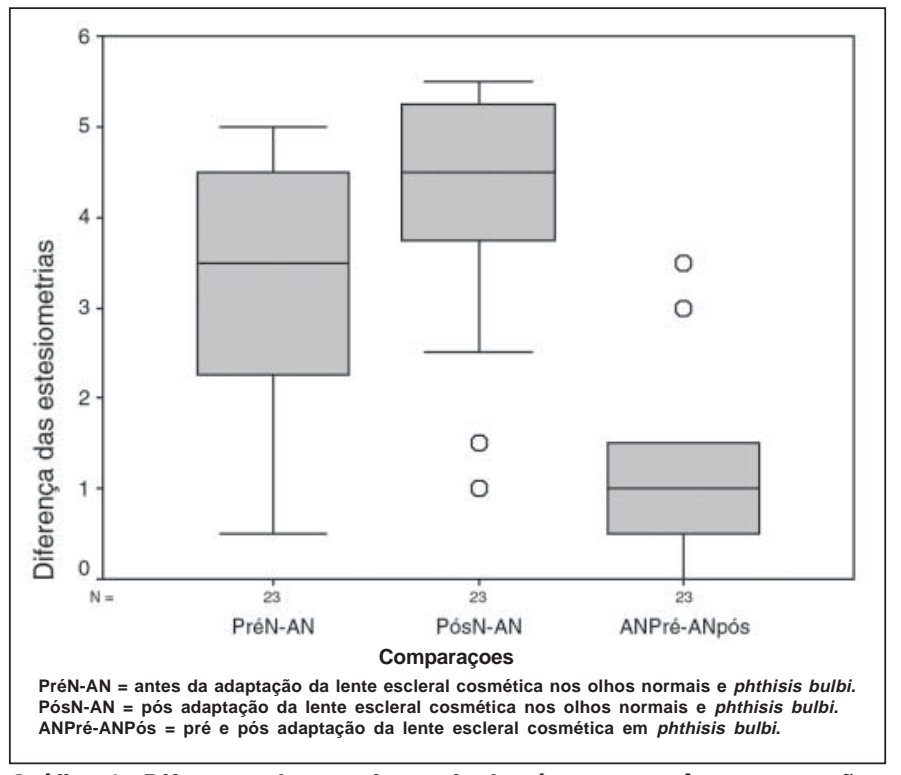

Gráfico 1 - Diferença da estesiometria da córnea nas três comparações

metabólica nas terminações nervosas corneanas causada pela baixa oxigenação(4)

Foram encontradas diferenças estatisticamente significantes nas 3 comparações realizadas entre os grupos deste estudo. As alterações provocadas pela LEC provavelmente são semelhantes às observadas nos usuários de LC.

Todos os pacientes deste estudo apresentaram uma boa tolerância ao uso da LEC. Talvez seja necessário um estudo com tempo de seguimento maior para se avaliar o efeito da LEC na sensibilidade tátil corneal em longo prazo.

A existência de phthisis bulbi leva o paciente a procurar um oftalmologista para melhorar sua estética, facilitando seu convívio social. Alguns casos necessitam de cirurgia para a retirada do bulbo ocular e reposição do volume perdido. Schellini et al. avaliaram 117 portadores de cavidade anoftálmica e constataram, nos implantes de vários materiais utiliza$\operatorname{dos}^{(12)}$. Assim sendo, nos casos de phthisis bulbi moderados a discretos, sem dor e com a superfície ocular sem ectopia, a adaptação de uma LEC pode retardar um procedimento cirúrgico. É importante ressaltar que há uma maior tolerância ao uso das LEC em phthisis bulbi devido à baixa sensibilidade tátil corneal, porém, sem este importante mecanismo de defesa da córnea, os usuários de LEC devem ser examinados periodicamente para se detectar qualquer com- plicação decorrente do uso de LEC ou pela evolução da phthisis bulbi.

\section{ABSTRACT}

Purpose: To compare corneal sensitivity between normal eyes and those whith phthisis bulbi and also to analyze the alterations of corneal sensitivity in phthisis bulbi induced by wearing ocular prosthesis. Methods: Prospective study of 23 patients with unilateral phthisis bulbi. Bilateral cornea sensitivity was evaluated using the Cochet-Bonnet esthesiometer before and after 3 months of wearing ocular prosthesis. Results: In all patients, corneal sensitivity of the eye with phthisis bulbi was lower than that of the normal eye (control). In $96 \%$ there was decrease of corneal sensitivity after adaptation of ocular prosthesis. Conclusion: After wearing ocular prosthesis, there is a reduction in corneal sensitivity in phthisis bulbi. The pathophysiology seems to be the same as that found in contact lens wearers.

Keywords: Cornea; Eye, artificial; Dermatitis, contact; Anoxia

\section{REFERÊNCIAS}

1. Schwartz DE. Corneal sensitivity in diabetics. Arch Ophthalmol. 1974;91(3):174-8.

2. Costa MS, Gallo MEN, Nery JAC, Benchimol E. Avaliação oftalmológica em hanseníase multibacilar. Arq Bras Oftalmol [periódico na Internet]. 1999 Nov-Dez [citado 2003 out 27];62(6):[ cerca de 4 p.]. Disponível em URL: http://www.abonet.com.br/abo/abo62601.htm

3. Millodot M. Effect of long-term wear of hard contact lenses on corneal sensitivity. Arch Ophthalmol. 1978;96(7):1225-7.

4. Velasco MJ, Bermúdez FJ, Romero J, Hita E. Variations in corneal sensitivity with hydrogel contact lenses. Acta Ophthalmol (Copenh). 1994;72(1):53-6.

5. Lawrenson JG, Ruskell GL. Investigation of limbal touch sensitivity using a Cochet-Bonnet aesthesiometer. Br J Ophthalmol. 1993;77(6):339-43.

6. Mathers WD, Jester JV, Lemp MA. Return of human corneal sensitivity after penetrating keratoplasty. Arch Ophthalmol.1988;106(2):210-1.

7. Gomes JAP, Haraguchi DKM, Zambrano DU, Villavicencio LI, Cunha MC, Freitas D. Punções do estroma anterior no tratamento da ceratopatia bolhosa. Arq Bras Oftalmol [periódico na Internet] 2000 Nov-Dez [citado 2003 out 27];63(2):[cerca de 6 p.]. Disponível em URL: http://www.abonet.com.br/abo/abo63202.htm

8. Rêgo MGB, Rodovalho AJM, Rocha AAA, Nassaralla Jr JJ, Nassaralla BRA. Sensibilidade corneana e secreção lacrimal após LASIK. Arq Bras Oftalmol [periódico na Internet]. 2003 Mar-Abr [citado 2003 out 27];66(2):[cerca de 8 p]. Disponível em URL: http://www.abonet.com.br/abo/662/abo66212.htm

9. Xu KP, Yagi Y, Tsubota K. Decrease in corneal sensitivity and change in tear function in dry eye. Cornea. 1996;15(3):235-9.

10. Cochet P, Bonnet R. L'esthesiometrie corneenne. Clin Ophthalmol. 1960;4:3-27.

11. Bussab WO, Morettin PA. Estatística básica. 4a. ed. São Paulo: Atual; 1987.

12. Schellini SA, Hoyama E, Padovani CR, Ferreira VL, Roça R. Complicações com uso de esferas não integráveis e integráveis na reconstrução da cavidade anoftálmica. Arq Bras Oftalmol. 2000;63(3):175-8. 